\title{
Do Sedentary Behaviors Modify the Health Status of Older Adults?
}

\author{
Elizabeth K. Lenz (Corresponding Author) \\ Kinesiology, Sport Studies, and Physical Education, The College at Brockport-SUNY \\ 350 New Campus Drive, Brockport, NY, USA, 14420 \\ Tel: 1-585-395-5266 E-mail: egrimm@brockport.edu \\ Ann M. Swartz \\ Department of Kinesiology, University of Wisconsin-Milwaukee \\ PO Box 413, Milwaukee, WI, 53201, USA \\ Tel: 1-414-229-4242 E-mail: aswartz@uwm.edu \\ Scott J. Strath \\ Department of Kinesiology, University of Wisconsin-Milwaukee \\ PO Box 413, Milwaukee, WI, 53201, USA \\ Tel: 1-414-229-3666 E-mail: sstrath@uwm.edu
}

Received: 21-11- 2013

Accepted: 28-12- 2013

Published: 31-01- 2014

doi:10.7575/aiac.ijkss.v.2n.1p.13

URL: http://dx.doi.org/10.7575/aiac.ijkss.v.2n.1p.13

\begin{abstract}
Evidence suggests sedentary behavior (SB) negatively impacts the health of adults but less is known about SB impact on older adult (OA) health. Seventy OA (73.4 \pm 6years) living in the southeast region of Wisconsin, United States of America (USA) completed three SB diaries and had risk factors associated with cardiovascular disease (CVD) assessed. Sedentary behaviors were quantified as time spent in sitting/lying activities. Pearson correlation coefficients, independent samples $t$-tests, and one-way ANOVA were performed to explore the relationship between SB and health. Older adults engaged in $620.3 \pm 91.2 \mathrm{mins} / \mathrm{d}$ of SB with television watching $(144.3 \pm 99.8 \mathrm{mins} / \mathrm{d})$ being the most prominent. Total SB and television watching were correlated to multiple risk factors for CVD $(r=-.241-.415, p=.009$ $.027)$ and these variables worsened as OA spent more time in those activities. Television watching was the only SB that increased across risk categories of $\operatorname{CVD}[F(2,67)=4.158, p=.020$, eta squared $=.11]$. These results suggest $\mathrm{SB}$, especially television watching to be related to risk factors of CVD in OA.
\end{abstract}

Keywords: Sedentary behavior, health, older adults, diary, cardiovascular disease risk factors

\section{Introduction}

It is well established that physical activity, across all age groups, is positively associated with a reduction in cardiovascular disease (CVD), stroke, hypertension, type II diabetes, osteoporosis, obesity, and some cancers, and improvements in anxiety and depression (Haskell et al., 2007; Nelson, Rejeski, \& Blaire, 2007). In older adults (OA) specifically, physical activity aids in the maintenance of physical function (Fielding et al., 2007), reduces fall risk and subsequent injury (Smulders et al., 2010) and is therapeutic for many chronic disease conditions (Hagberg, Montain, Martin, \& Ehsani, 1989; Janssen \& Joliffe, 2006; Kitzman, Brubaker, Morgan, Stewart, \& Little, 2010). However, it is well documented that a majority of OA do not engage in an appropriate amount of moderate to vigorous physical activity (MVPA) to reap the associated health benefits (Kruger \& Kohl, 2007; Troiano et al., 2007).

One factor that may largely contribute to a lack of engagement in health promoting MVPA in OA is an increased participation in total daily sedentary behavior (SB). Researchers have reported OA spending 11+ hours per day engaging in SB, specifically spending more than 50\% of their day in sitting behaviors (Matthews et al., 2008; Grimm et al., 2009; Seguin et al., 2012; Chau et al., 2013). In primarily younger and middle aged adults, a dose response relationship has been identified between time engaged in specific SB, such as watching television, and worsening health conditions independent of their time engaging in MVPA (i.e., impaired fasting blood glucose, obesity, elevated blood pressure, and cholesterol values), (Hu, Li, Colditz, Willett, \& Manson, 2003; Healy et al., 2007; Katzmarzyk, Church, Craig, \& Bouchard, 2009; Dunstan et al., 2010; Pinto Pereira, Ki, \& Power, 2012). Importantly, these health conditions are risk factors for the development of chronic disease conditions such as CVD and type II diabetes, which are highly prevalent in OA. Due to the existence of these known associations in younger age groups it is important to understand if this relationship continues with increasing age. In OA, there is a dearth of literature regarding specific SB in which they regularly participate as well as the extent to which these behaviors relate to their overall risk for chronic disease development. 
Recently a self-report SB diary was developed specifically for use in OA to further explore the SB of this population in detail (Grimm et al., 2009). The diary was designed to overcome a variety of limitations to objective SB assessment and provide additional information regarding the type and frequency of specific SB. Furthermore, this diary offers insight to information concerning enjoyment of certain behaviors, social support influences of SB, and whether OA engage in additional behaviors while being sedentary (e.g., eating).

Therefore, the purpose of the current study is to explore whether relationships exist between commonly self-reported SB specific to OA living in southeast regions of Wisconsin, USA and the health variables related to chronic disease development.

\section{Methods}

\subsection{Study Recruitment and Eligibility Criteria}

The participants were informed of this study through word of mouth, presentations at local senior centers, and the distribution of announcements at local businesses. Eligibility for involvement in this study included adults, 65-90 years of age, retired or no longer working in their primary profession, speaking English as their primary language, and the ability to ambulate without assisted walking devices or wheelchairs. All qualifying participants were scheduled for two laboratory visits at the University in which the study was conducted.

During the initial screening process all eligible OA were asked to describe their daily physical activity in an effort to gain a broad sample of individuals with a range in physical activity levels and potential variation in SB. Based upon their description of daily physical activity, their responses were grouped into three categories; participation in no leisure time physical activity (0-9 $\mathrm{min} / \mathrm{d}$ of MVPA), insufficient leisure time physical activity (10-29 $\mathrm{min} / \mathrm{d}$ of MVPA), and meeting the physical activity recommendations ( $\geq 30 \mathrm{mins} / \mathrm{d}$ of MVPA) (Nelson et. al., 2007). Based on their descriptions of self-reported physical activity, $27.1 \%$ engaged in no leisure time physical activity, $31.4 \%$ participated in an insufficient amount of MVPA, and $41.4 \%$ met the physical activity recommendations.

\subsection{Study Overview}

This was a cross-sectional study, designed to examine the relationship between SB and risk factors for chronic disease development in OA. During the first study visit participants read and signed a written informed consent document approved by the University's Institutional Review Board. Eligible participants then completed a health history questionnaire and had cardiovascular risk assessed. The specific cardiovascular risk assessments, discussed in detail below, were chosen because they are commonly used to detect level of risk for the development of CVD. Lastly participants were instructed to complete three SB diaries over a one-week timeframe prior to the second laboratory visit. When participants attended the second laboratory visit they returned the diaries and underwent additional cardiovascular health assessments. Subjects were informed of their results from all assessments at the end of the second visit.

\subsection{Study Methods}

\subsubsection{Visit One}

\subsubsection{Health History Questionnaire and Cardiovascular Risk Assessment}

All potential study participants were required to complete a health history questionnaire. This form inquired about general demographic information, present and past health history, and current use of medications. Once the health history questionnaire was completed participants underwent anthropometric assessments of height $(\mathrm{cm})$ and body mass $(\mathrm{kg})$. These were measured via calibrated physician's scale and stadiometer (Detecto, Kansas City, MO). Then body height was measured to the nearest $0.1 \mathrm{~cm}$ and body mass was measured to the nearest $0.01 \mathrm{~kg}$. From these values body mass index (BMI) was determined by dividing body mass $(\mathrm{kg})$ by body height in meters squared $\left(\mathrm{m}^{2}\right)$. Next, a constant tension tape measure was used to assess waist circumference $(\mathrm{cm})$ to the nearest $0.1 \mathrm{~cm}$. This measurement was taken around the narrowest part of the torso between the lowest rib and iliac crest according to standard procedures (Lohman, 1988). Blood pressure was assessed a minimum of two times in accordance with standard procedures to determine the risk factor hypertension (Pickering et al., 2005).

To evaluate the chronic disease risk factor of dyslipidemia a blood draw was taken to assess the following blood lipid profiles: total cholesterol, low-density and high-density lipoprotein cholesterols, plasma glucose, and triglycerides (National Cholesterol Education Program, (NCEP), 2012). Specific directions were provided to the participants prior to their first visit. All potential participants were instructed to fast or refrain from food and caffeinated beverages for 8-12 hours. An evacuated tube method was used to draw five $\mathrm{ml}$ of blood into a serum separator/preservative free tube (Vacuette Greiner Bio-One, Monroe NC). Immediately following the blood draw, the blood tube rested for 30 minutes and was centrifuged for 15 minutes. After the serum separated it was removed and stored in a refrigerator until analyzed by a certified laboratory, Dynacare Laboratories (Milwaukee, WI).

To analyze all blood lipids an OLYMPUS ${ }^{\circledR}$ System (Beckman Coulter, Inc.) was used. Total cholesterol assays were measured using a reagent that is a combination of esterase and oxidase. The coefficient of variation for the deamination of cholesterol was $1.8 \%$ for the low $(116.3 \mathrm{mg} / \mathrm{dL})$ to $2.1 \%$ for the high values $(2.1 \% \mathrm{mg} / \mathrm{dL})$. A series of coupled enzymatic reactions were used when triglycerides were measured and the absorbance change was proportional to the concentration of triglyceride. The coefficient of variation for this method was $2.5 \%$ for the low $(100 \mathrm{mg} / \mathrm{dL})$ to $1.2 \%$ for the high $(250 \mathrm{mg} / \mathrm{dL})$ values. To assess both the low-density and high-density lipoprotein cholesterols a two reagent homogenous system was used. The coefficients of variation were $3.39 \%$ for the low $(51.99 \mathrm{mg} / \mathrm{dL})$ to $3.84 \%$ for the 
high $(121.79 \mathrm{mg} / \mathrm{dL})$ low-density lipoprotein cholesterol. When high-density lipoprotein cholesterols were analyzed with the same methodology the coefficients of variation were $3.43 \%$ and $3.8 \%$ for the low $(38.59 \mathrm{mg} / \mathrm{dL})$ to high $(85.09$ $\mathrm{mg} / \mathrm{dL}$ ) values, respectively. The hexokinase G-6-PDH method was used to analyze glucose and the coefficient of variation for glucose was $1.4 \%$ for the low $(65 \mathrm{mg} / \mathrm{dL})$ to $1.2 \%$ for the high $(268 \mathrm{mg} / \mathrm{dL})$ value.

\subsubsection{Self-Report Sedentary Behavior Diary}

Each participant was provided both written and verbal instruction on the completion of three SB diaries (Grimm, Swartz, Harley, \& Strath, 2011). Specifically, the participants were asked to complete a 24-hour SB diary on three specific days during the following week, two weekdays and one weekend day (e.g., a diary was completed on Monday, Tuesday, Saturday for one participant and Wednesday, Friday, Sunday for another participant). The participants were randomly assigned which three days of the week the diaries were to be completed to ensure all days of the week were represented.

Participants were provided both written and verbal instructions that asked the participant to record a specific behavior they did during the majority of every half hour period throughout the day. Therefore, participants wrote down activities covering 48 half hour time slots for each diary completed. To further describe these behaviors, participants also had to circle whether they were sitting/lying or moving during the behavior, if they were alone during the behavior (yes/no), if they were eating during the behavior (yes/no), and to rank the enjoyment level of the behavior [1 (highly enjoyable) to 7 (least enjoyable)].

\subsubsection{Visit Two - Cardiovascular Risk Assessment}

All participants were instructed to wear minimal clothing and to be hydrated prior to the second visit. Weight for each participant was measured for a second time during this visit prior to undergoing body composition assessment via Dual Energy X-ray Absorptiometry (DXA; Lunar DPX-1, General Electric Lunar Corporation, Madison, WI). Total body fat percentage results were recorded.

\section{Data Analysis}

\subsection{Self-report Sedentary Behavior Diary}

All three SB diaries were examined for missing data. All activities were entered into a database and coded as a number (e.g., reading $=1$, watching television $=2$, reading $=3$, etc.). The circling of postural information was also coded for sitting/lying or standing (e.g., yes $=1$, no $=0$ ) and used to determine whether a specific behavior was truly sedentary.

\subsubsection{Statistical Analysis}

The statistical analysis was conducted with SPSS ${ }^{\circledR} 19.0$ for Windows (SPSS, Inc., Chicago, IL). Descriptive analysis is presented as mean \pm standard deviation for demographic information, health variables measured, and the time spent engaging in various self-reported SB. To better understand the relationship between health and specific SB, Pearson correlation coefficients were determined. Independent samples $t$-tests were conducted to determine whether gender differences arose in anthropometric, health variables, and time spent engaged in SB. One-way analysis of variance (ANOVA) were conducted to examine if there were differences in health across low, moderate, and high volumes (mins/d) of various SB and to examine whether SB related to risk for CVD. Significance for all analysis was set a priori, $p<0.05$.

\section{Results}

A total of 73 participants were recruited for the present study with 70 participants successfully completing the study (45 females and 25 males). All participants were retired for approximately $11.3 \pm 9.6$ years and most $(61.4 \%)$ volunteered for $3.2 \pm 5$ hours per week, were married (52.9\%), and/or lived with someone (61.4\%). Participant anthropometric and overall health characteristics are found in Table 1.

Table 1. Participant anthropometric and cardiovascular health characteristics.

\begin{tabular}{|c|c|c|c|}
\hline & $\operatorname{Men}(n=25)$ & Women $(n=45)$ & All $(N=70)$ \\
\hline Age (yrs) & $73.3 \pm 6.4$ & $73.4 \pm 5.8$ & $73.4 \pm 6$ \\
\hline $\mathrm{Ht}(\mathrm{cm})$ & $172.7 \pm 7.8 *$ & $161.6 \pm 5.4$ & $165.6 \pm 8.3$ \\
\hline Wt $(\mathrm{kg})$ & $81.3 \pm 13.2 *$ & $69.4 \pm 14$ & $73.7 \pm 14.8$ \\
\hline BMI $\left(\mathrm{kg} / \mathrm{m}^{2}\right)$ & $27.2 \pm 3.9$ & $36.6 \pm 5.3$ & $26.8 \pm 4.8$ \\
\hline $\mathrm{WC}(\mathrm{cm})$ & $92.7 \pm 10.7^{*}$ & $82.8 \pm 11.7$ & $86.4 \pm 12.2$ \\
\hline $\mathrm{SBP}(\mathrm{mmHg})$ & $123.7 \pm 10.3$ & $125 \pm 15.9$ & $124.5 \pm 13.9$ \\
\hline DBP (mmHg) & $79.8 \pm 9.6$ & $76.3 \pm 8.8$ & $77.6 \pm 9.2$ \\
\hline LDL (mg/dL) & $97.8 \pm 30$ & $104.5 \pm 25.4$ & $102.1 \pm 27.2$ \\
\hline HDL (mg/dL) & $56.5 \pm 15.7 *$ & $65.4 \pm 17.4$ & $62.2 \pm 17.2$ \\
\hline $\mathrm{TG}(\mathrm{mg} / \mathrm{dL})$ & $102.8 \pm 49.7$ & $123.4 \pm 60.2$ & $115.9 \pm 57.1$ \\
\hline $\mathrm{FBG}(\mathrm{mmol} / \mathrm{L})$ & $94 \pm 8.8$ & $91.6 \pm 8$ & $92.4 \pm 8.3$ \\
\hline $\mathrm{TC}(\mathrm{mg} / \mathrm{dL})$ & $166.9 \pm 43.8^{*}$ & $194.6 \pm 39.8$ & $184.6 \pm 37.7$ \\
\hline Body fat $(\%)$ & $25.3 \pm 7.6^{*}$ & $36.2 \pm 8.9$ & $32.3 \pm 9.9$ \\
\hline
\end{tabular}


Males were significantly taller, heavier, and had larger waist circumferences than women and had significantly lower body fat percentages and high-density lipoprotein cholesterol values. Almost all participants $(91.4 \%)$ were taking prescription medications, primarily controlling hypertension (45.7\%) and/or dyslipidemia (52.9\%). According to the American College of Sports Medicine Guidelines for determining CVD (American College of Sports Medicine, 2010), $11.6 \%, 58 \%$, and $30.4 \%$ of participants were categorized as low, moderate, and high risk for developing CVD, respectively. The percentages of all participant risk factors are in Table 2.

Table 2. Participant $(\mathrm{N}=70)$ risk factor frequencies for cardiovascular disease.

\begin{tabular}{lcccccc}
\hline & \multicolumn{2}{c}{ Men $(n=25)$} & \multicolumn{2}{c}{ Women $(n=45)$} & \multicolumn{2}{c}{ All $(N=70)$} \\
\hline Risk Factors for CVD & Yes & No & Yes & No & Yes & No \\
\hline Positive Risk Factor & & & & & & \\
$\quad$ Age & $100 \%$ & 0 & $100 \%$ & 0 & $100 \%$ & 0 \\
Family History & $32 \%$ & $68 \%$ & $17.8 \%$ & $82.2 \%$ & $22.9 \%$ & $77.1 \%$ \\
Obesity & $28 \%$ & $72 \%$ & $33.3 \%$ & $66.7 \%$ & $64.3 \%$ & 35.7 \\
Smoking & 0 & $100 \%$ & $4.4 \%$ & $95.6 \%$ & $2.9 \%$ & $97.1 \%$ \\
Sedentary & $36 \%$ & $64 \%$ & $51.1 \%$ & $48.9 \%$ & $45.7 \%$ & $54.3 \%$ \\
Hypertension & $56 \%$ & $44 \%$ & $40 \%$ & $60 \%$ & $45.7 \%$ & $54.3 \%$ \\
Dyslipidemia & $76 \%$ & $24 \%$ & $66.7 \%$ & $28.9 \%$ & $72.1 \%$ & $27.9 \%$ \\
$\quad$ Pre-diabetes & $32 \%$ & $68 \%$ & $15.6 \%$ & $80 \%$ & $21.4 \%$ & $75.7 \%$ \\
Negative Risk Factor & & & & & & \\
$\quad$ HDL & $32 \%$ & $68 \%$ & $51.1 \%$ & $44.4 \%$ & $45.6 \%$ & $54.4 \%$ \\
\hline
\end{tabular}

Upon examination of the SB diary, all participants reported $499.7 \pm 54.7 \mathrm{~min} / \mathrm{d}$ of sleeping at night, $940.3 \pm 54.7 \mathrm{mins} / \mathrm{d}$ of time awake, and, while awake, $620.3 \pm 91.2 \mathrm{mins} / \mathrm{d}$ engaging in SB. Men self-reported significantly more time being sedentary $(670.8 \pm 83.2 \mathrm{mins} / \mathrm{d})$ compared to women [592.2 $\pm 83.7 \mathrm{mins} / \mathrm{d} ; t(68)=3.771, p=.000$, eta squared $=.17]$. In general, participants reported approximately 16 sedentary behaviors. The time spent in each SB is presented in Figure 1.

During the hours of 7 a.m. to 10 p.m. these particular OA engaged in 10 or more SB with television watching $(144.3 \pm 99.8 \mathrm{mins} / \mathrm{d})$, reading $(102 \pm 80.8 \mathrm{mins} / \mathrm{d})$, eating meals $(96.1 \pm 39.6 \mathrm{mins} / \mathrm{d})$, using the computer $(68.3 \pm 69.3$ mins/d), and transportation activities $(67.1 \pm 46.1 \mathrm{mins} / \mathrm{d})$ being the most commonly reported. As seen in Figure 1., there were significant gender differences in time engaged in specific SB; women participated in significantly more transportation activities $(74.7 \pm 50.8 \mathrm{mins} / \mathrm{d})$ than $\mathrm{men}[53.6 \pm 32.6 \mathrm{mins} / \mathrm{d} ; t(68)=-2.106, p=.039$, eta squared $=.06]$, while men reported eating meals $(113.2 \pm 34.6 \mathrm{mins} / \mathrm{d})$ for a significantly longer duration than women $[86.7 \pm 39.3$ $\operatorname{mins} / \mathrm{d} ; t(68)=2.82, p=.006$, eta squared $=.10]$, and spending more time napping $(26.8 \pm 47.1 \mathrm{mins} / \mathrm{d})$ than women $[5.3 \pm 13.1 \mathrm{mins} / \mathrm{d} ; t(68)=2.877, p=.005$, eta squared $=.11]$.

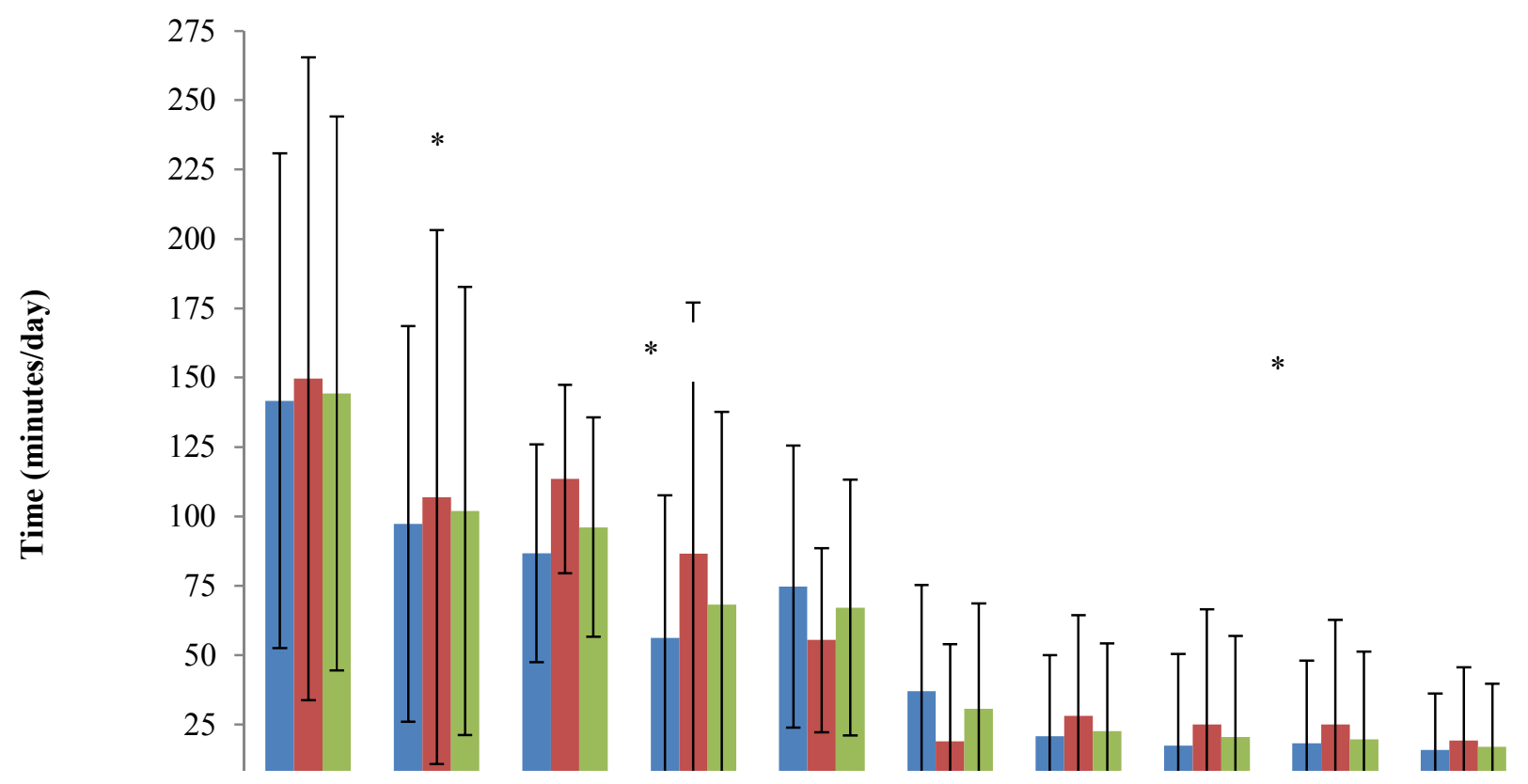

Figure 1. Differences in self-reported sedentary behaviors of men, women, and the entire group.

$*_{p}<.05$

Note. The data is presented in mean minutes per day \pm standard deviations.

$\mathrm{OA}$ in the present study engaged in five primary SB and these variables, including overall SB time, were further analyzed to determine if they related to cardiometabolic health. Pearson correlations coefficients demonstrating these relationships are found in Table 3. 
Table 3. Pearson correlations between cardiovascular health variables and sedentary behaviors for all participants $(\mathrm{N}=70)$.

\begin{tabular}{lllllll}
\hline Variables & TV & Reading & Eating & Computer & Transportation & Total SB \\
\hline Wt $(\mathrm{kg})$ & .206 & -.004 & -.064 & .221 & .141 & $.403^{* *}$ \\
BMI $\left(\mathrm{kg} / \mathrm{m}^{2}\right)$ & .213 & -.051 & -.168 & .114 & .216 & .213 \\
WC $(\mathrm{cm})$ & $.277^{*}$ & .002 & -.072 & .153 & .102 & $.415^{* *}$ \\
LDL $(\mathrm{mg} / \mathrm{dL})$ & -.175 & .074 & -.034 & .076 & -.005 & .051 \\
HDL $(\mathrm{mg} / \mathrm{dL})$ & $-.259^{*}$ & .089 & .037 & -.001 & .007 & $-.241^{*}$ \\
TG $(\mathrm{mg} / \mathrm{dL})$ & $.293^{*}$ & -.187 & -.236 & .149 & .042 & .165 \\
FBG $(\mathrm{mg} / \mathrm{dL})$ & .153 & $-.251^{*}$ & -.028 & -.003 & .007 & .186 \\
TC $(\mathrm{mg} / \mathrm{dL})$ & -.102 & .054 & -.105 & .109 & .016 & -.027 \\
Body Fat $(\%)$ & $.311^{*}$ & -.088 & $-.306^{*}$ & -.013 & .178 & .056 \\
SBP $(\mathrm{mmHg})$ & $.273^{*}$ & -.114 & -.065 & -.167 & -.079 & -.075 \\
DBP $(\mathrm{mmHg})$ & -.058 & .042 & .157 & -.034 & -.097 & -.032 \\
\hline
\end{tabular}

$*_{p}<.05, * * p<.01$

Note. $\mathrm{Wt}=$ weight; $\mathrm{BMI}=$ body mass index; $\mathrm{WC}=$ waist circumference; $\mathrm{LDL}=$ low density lipoprotein; $\mathrm{HDL}=$ high density lipoprotein; $\mathrm{TG}=$ triglycerides; $\mathrm{FBG}=$ fasting blood glucose; $\mathrm{TC}=$ total cholesterol; $\mathrm{SBP}=$ systolic blood pressure; $\mathrm{DBP}=$ diastolic blood pressure; $\mathrm{TV}=$ television; $\mathrm{SB}=$ sedentary behavior.

There are significant small to medium positive relationships between television viewing and waist circumference, triglycerides, percent body fat, and systolic blood pressure $(r=.273-.311, p=.009-.027)$ and a significant small negative correlation with high-density lipoprotein cholesterol $(r=-.259, p=.032)$. Correlations approached significance between television viewing and the variables body mass and BMI $(r=.206-.213, p=.077-.087)$. Significant small relationships were noted between time spent reading and fasting blood glucose $(r=-.251, p=.039)$ as well as eating and percent body fat $(r=-.306, p=.010)$. The specific SB of computer time and transportation were not significantly related to health variables. Total self-reported SB demonstrated significant medium positive relationships to body mass and waist circumference $(r=.403-.415, p=.000)$ and a significant negative small correlation with high-density lipoprotein cholesterol $(r=-.241, p=.046)$. The relationship between total reported SB and BMI approached significance $(r=.213, p=.077)$.

Due to limited findings observed between reading, eating meals, computer, transportation and health variables the remaining results section focuses on examining cardiovascular health variables related specifically to television viewing and overall time engaged in SB. A one-way ANOVA was conducted to determine if differences in health occurred across tertiles of television watching (low, $n=25: \leq 90 \mathrm{mins} / \mathrm{d}$, moderate, $n=23: 91-170 \mathrm{mins} / \mathrm{d}$, high, $n=22: \geq 171 \mathrm{mins} / \mathrm{d}$ ) and the results are found in Figure 2.

There were significant differences across television viewing times at the $p<.05$ level for body mass $[F(2,67)=3.509$, $p=.036$, eta squared $=.09]$, triglycerides $[F(2,66)=3.305, p=.043$, eta squared $=.09]$, percent body fat $[F(2,67)=$ $4.398, p=.016$, eta squared $=.12]$, and systolic blood pressure $[F(2,63)=6.792, p=.002$, eta squared $=.18]$, with BMI approaching significance $[F(2,67)=3.116, p=.051]$. Post-hoc comparisons using Tukey HSD test determined significant differences in body mass between the moderate $(69.2 \pm 12.9 \mathrm{~kg})$ and high TV groups $(80.1 \pm 15.5 \mathrm{~kg})$. Additionally significant differences resulting in the following variables between the low and high TV groups; triglycerides (low $=95.8 \pm 34 \mathrm{mg} / \mathrm{dL}$ and high $=137.7 \pm 73.6 \mathrm{mg} / \mathrm{dL})$, percent body fat $($ low $=28.6 \pm 10.4 \%$ and high $=$ $36.8 \pm 28.6 \%$ ), and systolic blood pressure (low $=122.7 \pm 10.2 \mathrm{mmHg}$ and high $=133 \pm 13.6 \mathrm{mmHg}$ ).

Figure 3 displays the results of a one-way ANOVA which was conducted to determine if differences in health variables occurred across three groups of overall SB time (low SB, $n=25: \leq 590 \mathrm{mins} / \mathrm{d}$, moderate $\mathrm{SB}, n=24: 591-670 \mathrm{mins} / \mathrm{d}$, high $\mathrm{SB}, n=21: \geq 671 \mathrm{mins} / \mathrm{d})$. There were significant differences at the $p<.05$ level for body mass $[F(2,67)=5.435, p=.006$, eta squared $=.14]$ and waist circumference $[F(2,67)=7.122, p=.002$, eta squared $=.18]$, with BMI approaching significance $[F(2,67)=2.705, p=.074]$. Post-hoc comparisons using Tukey HSD test determined significant differences in body mass between the low SB $(66.5 \pm 11.2 \mathrm{~kg})$ and moderate SB groups $(76.4 \pm 16.7 \mathrm{~kg})$ and between low SB and high SB groups $(79.2 \pm 13.4 \mathrm{~kg})$, waist circumference between low SB $(79.9 \pm 10.5 \mathrm{~cm})$ and moderate SB groups $(87.9 \pm 13.4 \mathrm{~cm})$ and low SB and high SB groups $(92.2 \pm 9.4 \mathrm{~cm})$. 


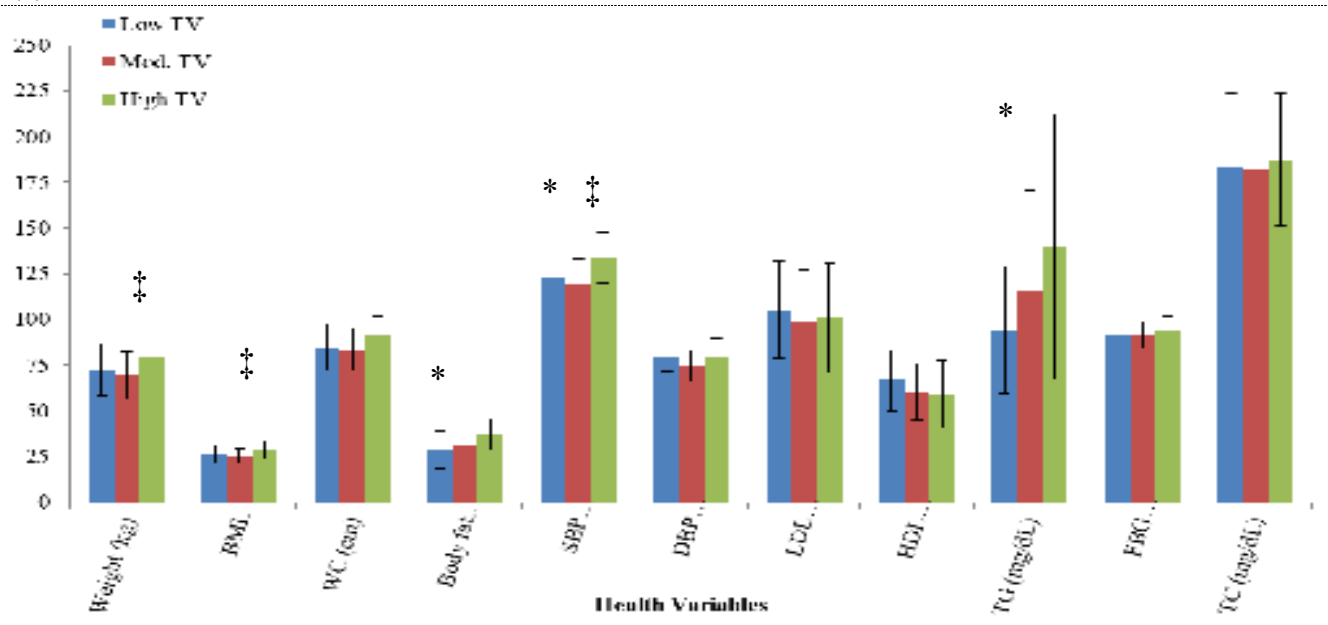

Figure 2. Differences in health variables across three television watching durations (mins/day)

$*=p<.05$ between low and high television for triglycerides $[F(2,69) 4.128, p=.020]$, percent body fat $[F(2,70) 4.774$, $p=.011]$, and systolic blood pressure $[F(2,66) 8.293, p=.001]$.

$\ddagger=p<.05$ between moderate and high television for Body weight $[F(2,70) 3.413, p=.039]$, body mass index $[F(2,70)$ 3.573, $p=.033$ ], and systolic blood pressure.

Notes. Low TV $=$ low television watching $(n=26 \leq 90 \mathrm{mins} / \mathrm{d})$; Mod TV $=$ moderate television watching $(n=24 ; 91$ $170 \mathrm{mins} / \mathrm{d})$, and high television watching $(n=23 ; \geq 171 \mathrm{mins} / \mathrm{d}) ; \mathrm{BMI}=$ body mass index; WC $=$ waist circumference; $\mathrm{SBP}=$ systolic blood pressure; $\mathrm{DBP}=$ diastolic blood pressure; $\mathrm{LDL}=$ low density lipoprotein; HDL=high density lipoprotein; $\mathrm{TG}=$ triglycerides; $\mathrm{FBG}=$ fasting blood glucose; $\mathrm{TC}=$ total cholesterol. Data is presented as mean $\pm \mathrm{SD}$.

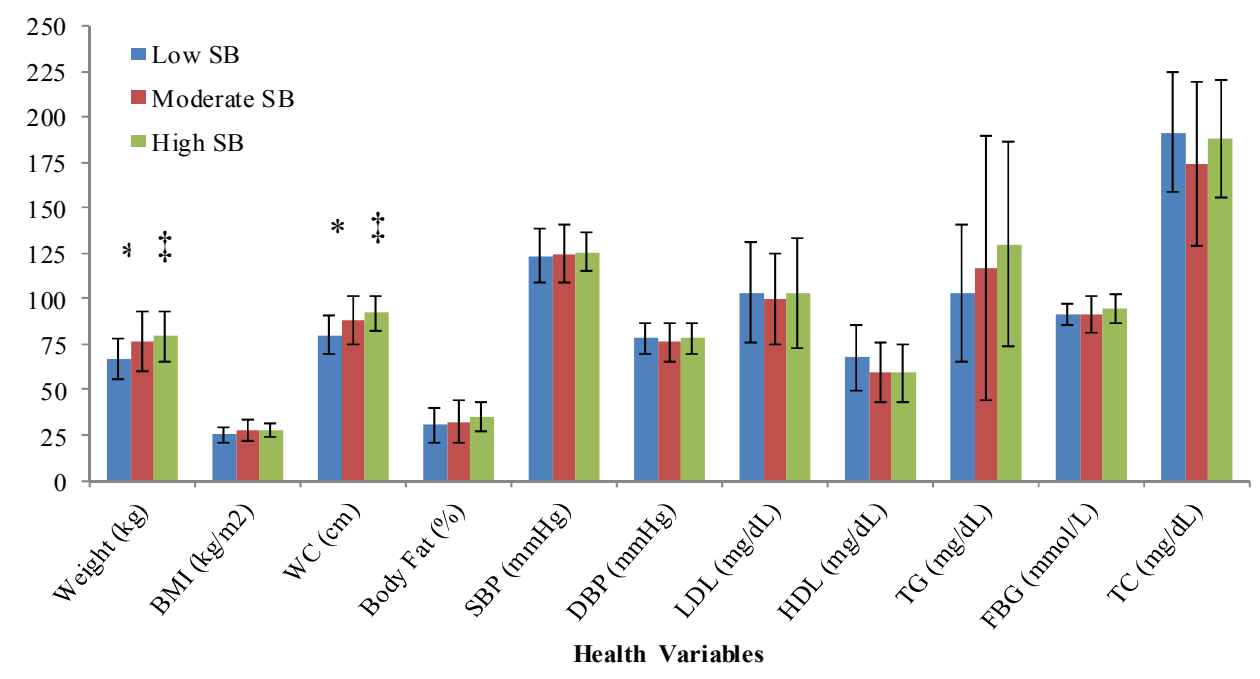

Figure 3. Differences in health variables across three durations of total sedentary behavior (mins/d) for the entire group $(\mathrm{N}=70)$

$*=p<.05$ between low and moderate SB for Body mass $[F(2,67) 5.435, \mathrm{p}=.006]$ and waist circumference $[\mathrm{F}(2,67)$ 7.122, $\mathrm{p}=.002]$.

$f=p<.05$ between low and high SB for Body mass and waist circumference.

Notes. SB = sedentary behavior; Low SB, n=25: $\leq 590$ minutes/day; Moderate SB, n=24: 591-670 minutes/day; High $\mathrm{SB}, \mathrm{n}=21: \geq 671$ minutes/day; $\mathrm{BMI}=$ body mass index; WC=waist circumference; $\mathrm{SBP}=$ systolic blood pressure; $\mathrm{DBP}=$ diastolic blood pressure; $\mathrm{LDL}=$ low density lipoprotein; $\mathrm{HDL}=$ high density lipoprotein; $\mathrm{TG}=$ triglycerides; $\mathrm{FBG}=$ fasting blood glucose; $\mathrm{TC}=$ total cholesterol. Data is presented as mean $\pm \mathrm{SD}$.

To determine whether individual SB and overall SB time differed across low $(n=8)$, moderate $(n=41)$, and high $(n=21)$ risk individuals for CVD a one-way ANOVA was performed and the results are displayed in Figure 4 . There were significant differences at the $p<.05$ level for television viewing $[F(2,67)=4.158, p=.020$, eta squared $=.11]$. Post-hoc comparisons using Tukey HSD test determined significant differences suggesting low risk individuals spend significantly less time watching television $(58.8 \pm 48.8 \mathrm{mins} / \mathrm{d})$ than high risk individuals $(172.9 \pm 116.3 \mathrm{mins} / \mathrm{d})$. A difference in television viewing time between low and moderate risk individuals (146.3 \pm 90 mins/d) approached 
significance, $p=.053$. There were no significant differences in time spent with reading, transportation, computer, eating meals, and overall SB across CVD risk $(\mathrm{p}=.148-.744)$.

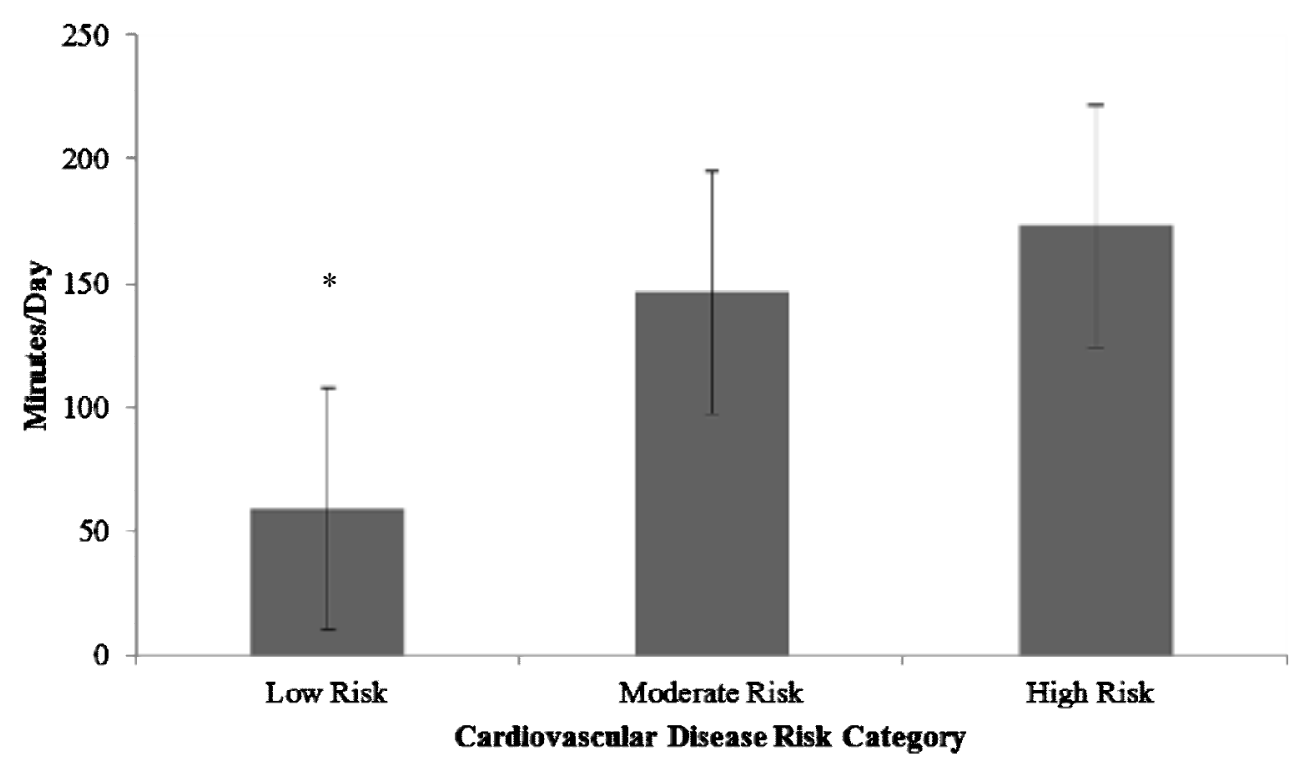

Figure 4. Differences in television watching time across low $(n=8)$, moderate $(n=41)$, and high $(n=21)$ risk categories for Cardiovascular Disease $(N=70)$

$*=p<.05$ between low and high risk categories for Cardiovascular Disease $[F(2,67) 4.158, p=.020]$.

Notes: Participants were determined low risk if they had $<2$ positive risk factors for cardiovascular disease, moderate risk if they had $\geq 2$ positive risk factors for cardiovascular disease, and high risk if they had known cardiovascular, pulmonary, or metabolic disease and/or major signs/symptoms suggestive of cardiovascular, pulmonary, or metabolic disease according to the American College of Sports Medicine.

\section{Discussion}

The present study uses self-report diaries to explore the specific SB of OA as well as investigate whether specific SB or the total time engaged in SB is negatively associated with health. The general findings suggest OA engaged in approximately 16 different SB. The most frequent reported SB were watching television, using the computer, eating meals, reading, and sitting in a vehicle for transportation. The specific SB of television watching was positively associated with approximately four risk factors for CVD: dyslipidemia determined by triglycerides and high-density lipoproteins, obesity established by waist circumference and percent body fat, and hypertension relating to systolic blood pressure. Total SB was associated with only two risk factors associated with CVD: obesity measured by waist circumference and dyslipidemia determined by high-density lipoproteins. These are important findings suggesting that time spent watching television as well as overall time engaged in SB may contribute to positive risk factors for CVD in OA.

The OA in the current study engaged in SB for approximately $63 \%$ of the time they reported being awake. There was commonality between the reported behaviors in the present study and those described in the American Time Use Survey (Bureau of Labor Statistics, 2008) in which OA were asked to describe the activities they engaged in on an average day. The survey found that OA spend a majority of the day watching television (54.5\%), reading (12.5\%), socializing with others $(11 \%)$, and relaxing (9\%), which could be assumed SB by definition (Owen, Leslie, Salmon, \& Fotheringham, 2000; BLS, 2008; Pate, O’Neill, Lobelo, 2008). After examining SB diaries, watching television was also gleaned to be the most engaged in behavior, however OA in the present study only watched approximately 2.5 hours per day (15.6\% of their awake-time). Reading was the second most cited behavior and OA reported spending about $10.6 \%$ of their awake-hours engaged in this behavior, which was similar to the American Time Use Survey. Other common behaviors like socializing and relaxing were reported less frequently in the present study, with only $3 \%$ of time awake spent socializing in a sedentary fashion and $<1 \%$ of the day relaxing. These differences are most likely due to the specificity of the SB diaries and the question format of the American Time Use Survey. Despite methodology differences both study results suggest OA engaged in SB for a large portion of their day, especially in the form of watching television.

In younger and middle-aged populations, total time engaged in SB measured objectively with accelerometry as well as time watching television when used as a surrogate measure of SB is observed to be related to a number of health conditions such as impaired fasting blood glucose (Dunstan et al., 2004; Dunstan et al., 2007; Healy et al., 2008; Chang et al., 2008), obesity when determined by BMI, waist circumference, skinfold assessment, and dual x-ray absorptiometry (Tucker \& Friedman, 1989; Tucker \& Bagwell, 1991; Ching et al., 1996; Fung et al., 2000; Hu et al., 2003; Jakes et al., 2003; Swartz et al., 2012), elevated blood pressure (Thorp, Healy, \& Owen, 2009) and blood lipids (i.e., triglycerides and high-density lipoproteins) (Healy et al., 2007; Chang et al., 2008; Healy et al., 2008; Thorp et al., 2009). The present study observed similar findings in OA; television viewing was related to elevated triglyceride levels 
and lower high-density lipoproteins, excess body fat and increased waist circumference and elevated systolic blood pressure.

Total SB was positively related to body mass and waist circumference and as OA engaged in $\leq 9.8$ hours per day to $\geq 11$ hours per day of $\mathrm{SB}$, body mass and waist circumferences increased $12.7 \mathrm{~kg}$ and $12 \mathrm{~cm}$, respectively. Television watching negatively impacted more health variables (e.g., increased body mass, triglycerides, percent body fat, systolic blood pressure, and lower high-density lipoprotein cholesterol) than total SB, even though television viewing represented only a small fraction of the total reported $\mathrm{SB}, \leq 2.5$ hours per day. These relationships were further supported when health variables were compared across low, moderate, and high volumes of television viewing (low, $\leq 1.5$ hours per day to high, $\geq 2.9$ hours per day). There were significant increases in body mass $(11 \mathrm{~kg})$, triglyceride values $(42 \mathrm{mg} / \mathrm{dL})$, body fat percentage $(8.2 \%)$, and systolic blood pressure $(10 \mathrm{mmHg})$ across these three volumes of television viewing.

Overweight or obesity and hypertension are the most prevalent risk factors for CVD in OA and the present study observed total SB and television viewing to relate to both of these risk factors (National Center for Health Statistics, 2003). The additional relationships between television time and various blood lipids and systolic blood pressure is concerning as they are also positive risk factors for CVD. The differences in these variables across volumes of SB and television time are enough to move OA out of optimal/desirable healthy ranges into abnormal/undesirable categories potentially contributing to the progression from low and/or moderate risk to high risk for the development of CVD. These findings are further supported when time watching television was compared across the OA categorized as low, moderate, and high risk for CVD. The low risk OA watched approximately two hours less television than those categorized as being high risk for CVD. Even though significance was not observed $(\mathrm{p}=.053)$ there was approximately an 88 minute difference in time watching television between the low and moderate risk OA. These findings are of paramount importance, with CVD representing one of the leading causes of death for this population (Heron, 2010).

The overall findings of this study suggest that watching television and/or engaging in large amounts of total SB is negatively associated with health of OA. To date the cause and effect relationships between these behaviors and health have not been determined. Some postulated mechanisms regarding SB and health suggest the reduced muscle activity accompanying SB may lead to the development of dyslipidemia due to lack of muscle contractions altering lipoprotein lipase activity and the blunting of GLUT 4 activity, reducing the ability to bring glucose effectively into the muscle (Bey \& Hamilton, 2003; Zderic \& Hamilton, 2006; Hamilton, Hamilton, Zderic, 2007). The adverse health changes relating to television watching is hypothesized to occur because it is one of the lowest energy expending activities compared to other SB (e.g., writing and playing games) (Ainsworth et al., 2000). Additionally people tend to engage in other behaviors like eating while watching television, often consuming more than average amounts of food and/or higher calorically dense foods that may lead to weight gain and/or increases in body fat (Hu et al., 2003). However, more research is necessary to determine the cause of these physiological changes related to SB in OA.

The present study adds to the current literature examining SB and its impact on health in OA. Individual SB typically engaged in are portrayed as well as whether relationships exist with an individual's risk factors for the development of CVD. These findings contribute to the need for more interventions to reduce total SB in OA through decreasing time watching television. The present study is not without limitations. The study was a cross-sectional examination of the specific types of SB and how they may impact the health of OA. As a result the cause and effect relationship between television and total SB impact cannot be established. The methodology of assessing SB was self-report and estimations of time spent in these behaviors are dependent of the participant adhering to the diary procedures.

\section{Conclusion}

The results of the present study provide a description of commonly performed SB of OA and suggest total SB and the specific act of television watching to be influential on multiple health variables relating to OA overall risk of CVD. Because CVD is highly prevalent in the OA population further investigation on the impact of these behaviors and health is warranted. Interventions designed to reduce overall SB and especially television watching may lead to positive changes in OA health.

\section{References}

Ainsworth, B. E., Haskell, W. L., Whitt, M. C., Irwin, M. I., Swartz, A. M., Strath, S. J. O’Brien, W. L...Leon, A. S. (2000). Compendium of physical activities: classification of energy costs of human physical activities. Medicine and Science in Sports and Exercise, 32(9), 498-504.

Thompson, W. R., Gordon, N. F., \& Pescatello, L. S. (Eds.). (2009). ACSM's Guidelines for Exercise Testing and

Prescription (8 ed.). Philadelphia, PA: Lippincott, Williams, and Wilkins.

Bey, L, \& Hamilton M. T. (2003). Suppression of skeletal muscle lipoprotein lipase activity during physical inactivity: a molecular reason to maintain low-density activity. Journal of Physiology, 551.2, 673-683. doi: 10.1113/jphysiol.2003.045591.

Bureau of Labor and Statistics. (2008). Leisure Time of Individuals Age 65 and Older: Employed versus Unemployed. Retrieved from http://www.bls.gov/tus/charts/older.htm. 
Chang, P-C., Li, T-C., Wu, M-T., Liu, C-S., Li, C-I., Lin, W-Y....Lin, C-C. (2008). Association between television viewing nad the risk of metabolic syndrome in a community-based population. BMC Public Health, 8, 198. doi: 10.1186/1471-2458-8-193.

Chau, J. Y., Grunseit, A. C., Chey, T., Stamatakis, E., Brown, W. J., Matthews, C. E....Plowe, H. P. (2013). Daily sitting time and all-cause mortality: a meta-analysis. PloS One, 8(11). Doi: 10.1371/journal.pone.0080000.

Ching, P., Willett, W. C., Rimm EB, Colditz, G. A., Gortmaker, S. L., \&. Stampfer,M. J. (1996). Activity level and risk of overweight in male health professionals. American Journal of Public Health, 86, 25-28.

Dunstan, D. W., Salmon, J., Owen, N., Armstrong, T., Zimmet, P. Z., Welborn, T. A., Cameron, A. J....Shaw, J. E. (2004). Physical activity and television viewing in relation to risk of undiagnosed abnormal glucose metabolism in adults. Diabetes Care, 27, 2603-2609.

Dunstan, D. W., Salmon, J., Healy, G. N., Shaw, J. E., Jolley, D., Zimmet, P. Z., \& Owen, N. (2007). Association of television viewing with fasting and 2 -h postchallenge plasma glucose levels in adults without diagnosed diabetes. Diabetes Care, 30(3), 516-522.

Dunstan, D. W., Barr, E. L. M., Healy, G. N., Salmon, J., Shaw, J. E., Balkau, B.,...Zimmet, P. Z. (2010). Television viewing time and mortality.The Australian diabetes, obesity, and lifestyle study (Ausdiab). Circulation, 121, 384-391. doi: 10.1161/CIRCULATIONAHA.109.894824

Fielding, R. A., Katula, J., Miller, M. E., Abbott-Pillola, K., Jordon, A., Blynn, N. W.,... Rejeski, W. J. (2007). Activity adherence and physical function in older adults with functional limitations. Medicine and Science in Sports and Exercise. 39(11), 1997-2004. doi:10.1249/mss.0b013e318145348d.

Fung, T. T., Hu, F. B., Spiegelman, D., Tofler, G. H., Willett, W. C., \& Rimm, E. B. (2000). Leisure-time physical activity, television watching, and plasma biomarkers of obesity and cardiovascular disease risk. American Journal of Epidemiology, 152(12), 1171-1178.

Grimm, E. G., Strath, S. J., Swartz, A. M., Miller, N. E., Gennuso, K. P., Dondzila, C. J., \& Sweere, K. M. (2009). Objective measurement of sedentary and active behaviors in older adults [Abstract]. Medicine and Science in Sports and Exercise. 41(Suppl 15), 379.

Grimm, E. K., Harley, A., Mathiowetz, N. A., Swartz, A. M., \& Strath, S. J. (2011). The exploration of sedentary behavior in older adults [Abstract]. Medicine and Science in Sports and Exercise. 43(Suppl 15), 539.

Hagberg, J. M., Montain, S. J., Martin, W. H., \& Ehasani, A. A. (1989). Effect of exercise training in 60-69 year old persons with essential hypertension. American Journal of Cardiology.=, 64, 348-353.

Hamilton, M. T., Hamilton D. B., \& Zderic, T. W. (2007). Role of low energy expenditure and sitting in obesity, metabolic syndrome, type 2 diabetes, and cardiovascular disease. Diabetes, 56, 2655-2667. doi: 10.2337/db07-0882

Healy, G. N., Dunstan, D. W., Salmon, J., Cerin, E., Shaw, J. E., Zimmet, P. Z., \& Owen, N. (2007). Objectively measured light-intensity physical activity is independently associated with 2-h plasma glucose. Diabetes Care, 30,13841389. doi: $10.2337 / \mathrm{dc} 07-0114$.

Healy, G. N., Wijndaele, K., Dunstan, D. W., Shaw, J. E., Salmon, J., Zimmet, P. Z., \& Owen, N.et al. (2008). Objectively measured sedentary time, physical activity, and metabolic risk: the Australian Diabetes, Obesity and Lifestyle Study (AusDiab). Diabetes Care, 31, 369-371. doi: 10.2337/dc07-1795.

Heron, M. (2010). Deaths: Leading causes for 2006. National vital statistics reports. 58(14). Hyattsville, MD: National Center for Health Statistics. Retrieved from http://www.cdc.gov/nchs/data/nvsr/nvsr58/nvsr58_14.pdf.

Hu, F. B., Li, T. Y., Colditz, G. A., Willett, W. C., \& Manson, J. E. (2003). Television watching and other sedentary behaviors in relation to risk of obesity and type 2 diabetes mellitus in women. Journal of the American Medical Association, 289(14), 1785-1791.

Jakes, R. W., Day, N. E., Khaw, K. T., Luben, R., Oakes, S., Welch, A., Bingham, S., \& Wareham, N. J. (2003). TV viewing and low participation in vigorous recreation are independently assoc with obesity and markers of cardiovascular disease risk: EPIC-Norfolk population-based study. European Journal of Clinical Nutrition, 57(9), 10891096. doi:10.1038/sj.ejcn.1601648.

Janssen, I., \& Jolliffe, C. (2006). Influence of physical activity on mortality in elderly with coronary heart disease. Medicine and Science in Sports and Exercise, 38, 418-423. doi: 10.1249/01.mss/0000191185.58467.be.

Katzmarzyk, P. T., Church, T. S., Craig, C. L., \& Bouchard, C. (2009). Sitting time and mortality from all causes, cardiovascular disease, and cancer. Medicine and Science in Sports and Exercise, 41(5), 998-1005. doi: 10.1249/MSS.0b013e3181930355.

Kitzman, D. W., Brubaker, P. H., Morgan, T. M., Stewart, K. P, \& Little, W. C. (2010). Exercise training in older patients with heart failure and preserved ejection fraction: a randomized, controlled, single-blind trial. Circulation: Heart Failure, 3(6), 659-667. doi: 10.1161/CIRCHEARTFAILURE.110.958785.

Kruger, J., \& Kohl, H. W. (2007). Prevalence of regular physical activity among adults, United States, 2001 and 2005. MMWR Morbidity Mortality Weekly Report, 56, 1209-1212. doi:10.1038/sj.ijo.0803386. 
Lohman, T. G. (1988). In A. F. Roche, S. B., Heymsfield, \& T. G., Lohman (Eds.), Anthropometric standardization reference manual. Champaign, IL: Human Kinetics.

Matthews, C. E., Chen, K. Y., Freedson, P. S., Buchowski, M. S., Beech, B. M., Pate, R. R., \& Troiano, R. P. (2008). Amount of time spent in sedentary behaviors in the United States, 2003-2004. American Journal of Epidemiology, 167(7), 875-881. doi: 10.1093/aje/kwm390.

National Center for Health Statistics. (2010). Health, United States, 2009: With special feature

on Medical Technology. Hayattsville, MD. Retrieved from http://www.cdc.gov/nchs/data/hus/hus09.pdf .

National Cholesterol Education Program. (2012). Third report of the national cholesterol education program (NCEP) expert panel on detection evaluation, and treatment of high blood cholesterol in adults (Adult Treatment Panel III).

Final Report. (National Institute of Health Publication No. 02-5215). Retrieved from http://www.nhlbi.nih.gov/guidelines/cholesterol/atp3full.pdf.

Nelson, M. E., Rejeski, W. J., \& Blair, S. N. (2007). Physical activity and public health in older adults: recommendation from the American College of Sports Medicine and the American Heart Association. Medicine and Science in Sports and Exercise, 39, 1435-1445. doi: 10.1249/mss.0b013e3180616aa2.

Owen, N., Leslie, E., Salmon J, \& Fotheringham, M. J. (2000). Environmental determinants of physical activity and sedentary behavior. Exercise and Sport Sciences Review, 28(4), 153-158.

Pate, R. R., O'Neill, J. R., \& Lobelo, F. (2008). The evolving definition of "sedentary". Exercise and Sport Science Review, 36(4), 173-178. doi: 10.1097/JES.0b013e3181877d1a.

Pickering, T. G., Hall, J. E., Appel, L. J., Falkner, B. E., Graves, J., Hill, M. N...Roccella, E. J. (2005). Recommendations for blood pressure measurement in humans and experimental animals: Part 1: blood pressure measurement in humans: a statement for professionals from the Subcommittee of Professional and Public Education of the American Heart Association Council on High Blood Pressure Research. Hypertension, 45(1), 142-161. doi: 10.1161/01.HYP.0000150859.47929.8e.

Pinto Pereira, S. M., Ki, M., \& Power, C. (2012). Sedentary behaviours and biomarkers for cardiovascular disease and diabetes in mid-life: the role of television viewing and sitting at work. PLoS One, 7(2). doi:10.1371/journal/pone.0031132.

Seguin, R., LaMonte, M., Tinker, L., Liu, J., Woods, N., Michael, Y. L.... Lacroix, A. Z. (2012). Sedentary behavior and physical function decline in older women: findings from the women's health initiative. Journal of Aging Research, Advance online publication. doi: 10.1155/2012/271589.

Smulders, E., Weerdesteyn, V., Groen, B. E., Duysens, J., Eijsbouts, A., Laan, R., \& van Lankveld, W. (2010). Efficacy of short multidisciplinary falls prevention program for elderly persons with osteoperosis. Archives of Physical Medicine and Rehabilitation, 91(11), 1701-1711. doi:10.1016/j.apmr.2010.08.004.

Swartz, A. M., Tarima, S., Miller, N. E., Hart, T. L., Grimm, E. K., Rote, A. E., \& Strath, S. J. (2012). Prediction of body fat in older adults by time spent in sedentary behavior. Journal of Aging and Physical Activity, 20 (3), $332-344$. Epub 2011 Dec 20.

Thorp, A. A., Healy, G. N., \& Owen, N. (2009). Deleterious associations of sitting time and television viewing with cardio-metabolic risk biomarkers: AusDiab 2004-2005. Diabetes Care, 33, 327-334. doi: 10.2337/dc09-0493.

Troiano, R. P., Berrigan, D., Dodd, K. W., Masse, L. C., Tilert, T., \& McDowell, M. (2007). Physical activity in the United States measured by accelerometer. Medicine and Science in Sports and Exercise, 40(1), 181-188. doi: 10.1249/mss.0b013e31815a5lb3.

Tucker ,L. A. \& Friedman, G. M. (1989). Television viewing and obesity in adults males. American Journal of Public Health, 79, 516-518.

Tucker, L. A. \& Bagwell, M. (1991). Television viewing and obesity in adult females, American Journal of Public Health, 81, 908-911.

Zderic, T. W \& Hamilton, M. T. (2006). Physical inactivity amplifies the sensitivity of skeletal muscle to the lipidinduced down regulation of lipoprotein lipase activity. Journal of Applied Physiology, 100, $249-257$. doi:10.1152/japplphysiol.00925.2005. 